Document downloaded from:

http://hdl.handle.net/10251/140834

This paper must be cited as:

Acedo Rodríguez, L.; Burgos-Simon, C.; Hidalgo, J.; Sánchez-Alonso, V.; Villanueva Micó, RJ.; Villanueva-Oller, J. (2018). Calibrating a large network model describing the transmission dynamics of the human papillomavirus (HPV) using a Particle Swarm Optimization (PSO) algorithm in a distributed computing environment. International Journal of High Performance Computing Applications. 32(5):721-728.

https://doi.org/10.1177/1094342017697862

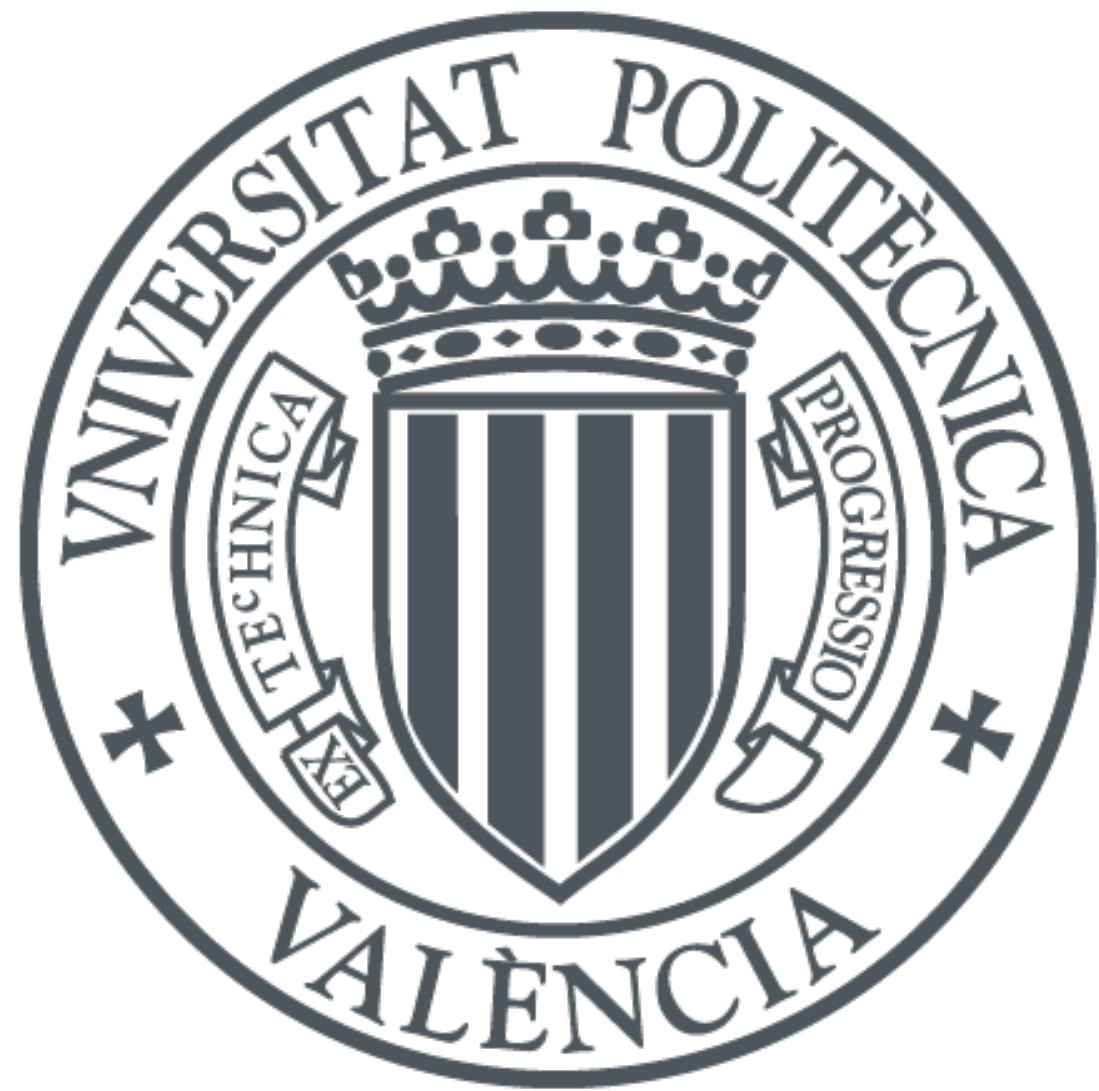

The final publication is available at

https://doi.org/10.1177/1094342017697862

Copyright SAGE Publications

Additional Information 


\title{
Calibrating a large network model describing the transmission dynamics of the human papillomavirus (HPV) using a Particle Swarm Optimization (PSO) algorithm in a distributed computing environment
}

Journal Title

$\mathrm{XX}(\mathrm{X}): 2-14$

(C) The Author(s) 0000

Reprints and permission:

sagepub.co.uk/journalsPermissions.nav

DOI: $10.1177 /$ ToBeAssigned www.sagepub.com

@SAGE

\section{Luis Acedo ${ }^{1}$, Clara Burgos ${ }^{1}$, José-Ignacio Hidalgo ${ }^{2}$, Víctor Sánchez-Alonso ${ }^{1}$, Rafael-Jacinto Villanueva ${ }^{1}$ and Javier Villanueva-Oller ${ }^{3}$}

\begin{abstract}
Working in large networks applied to epidemiological-type models has led us to design a simple but effective computed distributed environment to perform a large amount of model simulations in a reasonable time in order to study the behavior of these models and to calibrate them. Finding the model parameters that best fit the available data in the designed distributed computing environment becomes a challenge and it is necessary to implement reliable algorithms for model calibration. In this paper, we have adapted the random PSO algorithm to our distributed computing environment to be applied to the calibration of a Papillomavirus transmission dynamics model on a lifetime sexual partners network. And we have obtained a good fitting saving time and calculations compared with the exhaustive searching strategy we have been using so far.
\end{abstract}

\section{Keywords}

Network models, Distributed computing paradigm, Model calibration, Particle Swarm Optimization, Human Papillomavirus

Prepared using sagej.cls [Version: 2016/06/24 v1.10] 


\section{Introduction}

Sexually Transmitted Diseases (STD) have been a major public health threat for a long time in human history. Modern concerns about STD began with the pandemic of syphilis which spread over Europe in the early sixteenth century.

Human Papillomavirus (HPV) is the most common STD. It is transmitted via vaginal, anal, or oral sex with someone who has the virus [1]. Persistent HPV infections with genotypes 16 and 18 are responsible for about $70 \%$ of all cervical cancer, with $40-85 \%$ of other anogenital cancers and also $16-28 \%$ of the head and neck cancers. Furthermore, HPV is a cause of other non malignant diseases, to mention genotypes 6 and 11 cause about $90 \%$ of anogenital warts, and secondarily juvenile onset of recurrent respiratory papillomatosis [2].

Most of the modeling approaches to STD in general and HPV in particular are done using classical models where the hypothesis of homogeneous mixing (everybody can transmit a disease to everybody) is implicitly assumed. However, when STDs are considered, homogeneous mixing is not a reasonable hypothesis and consequences of this assumption can be seen, for instance, in that the effects of vaccination schedules against HPV have been detected much sooner than what the models predicted [3].

Therefore, the structure and properties of networks of sexual contacts in human populations and the building of reliable sexual partner networks, as in [4], is a public health topic of key interest in connection with the spread of STD. However, this problem has received scarce attention in the world of computational modeling and the modeling of STD epidemiology is usually based upon theoretical proposals in terms of the network structure.

In the last years, we have been working on modeling the dynamics of several phenomena using large random networks $[5,6,7]$ and we know that, under this approach, it is necessary to perform a lot of simulations for model calibration. To do so, a distributed computing environment called Sisifo [8] was developed [9]. Using this environment we were able to find, using exhaustive searching, model parameters that made the model output close to given data, that is, calibration.

\footnotetext{
${ }^{1}$ Instituto Universitario de Matemática Mulstidiciplinar, Universitat Politècnica de Valéncia, Valencia, Spain

${ }^{2}$ Departamento de Arquitectura de Computadores y Automática, Universidad Complutense de Madrid, Madrid, Spain

${ }^{3}$ Departamento de Ciencias de la Computación, Arquitectura de Computadores, Lenguajes y Sistemas Informáticos, Estadística e Investigación Operativa, Universidad Rey Juan Carlos, Móstoles (Madrid), Spain
}

Corresponding author:

Instituto Universitario de Matemática Mulstidiciplinar, Edificio 8G, piso 2, Universitat Politècnica de Valéncia, Valéncia, Spain

Email: rjvillan@imm.upv.es

Prepared using sagej.cls 
The next step was to find out how to reduce the number of simulations studying the inclusion of optimization algorithms. In [10] we presented an attempt with small networks and in an only computer.

Then, in this paper, we describe how to adapt the optimization algorithm named Particle Swarm Optimization (PSO) in the distributed computing environment Sisifo to calibrate a large network of lifetime sexual partners (LSP) where we want to study the transmission dynamics of two types of HPV: the high and the low risk. High risk HPV gathers the types of HPV related to the apparition of precancerous lesions or cancers. Low risk HPV types are the ones that can develop genital warts.

This paper is organized as follows. In Section 2 we give an overview of the LSP network building and describe the transmission dynamics of both types of HPV on LSP networks. In Section 3, we summarize the functioning of the distributed computing environment Sisifo. Also, we describe how to incorporate and adapt the PSO algorithm to this environment. In Section 4 we present the results and, finally, in Section 5, conclusions are discussed.

\section{HPV network model building}

Here we give a general review of the method presented in [4] where an algorithm to build LSP networks consistent with the distribution of the number of partners for both males and females reported in the Health and Sexual Habits Survey in Spain [11], has been described. Then, on the LSP networks, we will define the transmission dynamics of high and low risk HPV.

First, we consider the population of the Community of Valencia (Spain) [12] and its demographic structure [13] given in Figure 1, where we show the number of men and woman per age.

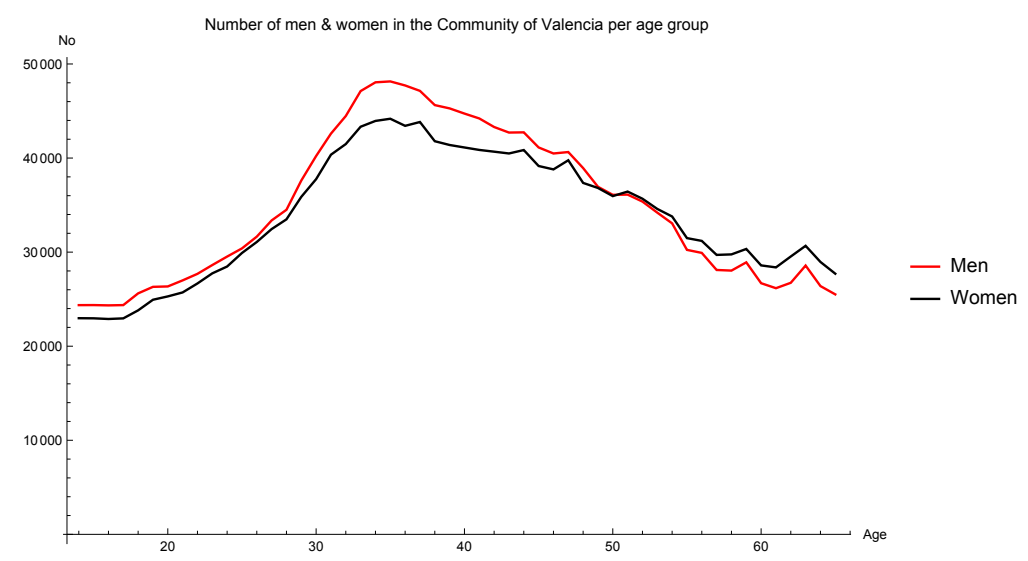

Figure 1. Number of men and woman in the Community of Valencia per age (year 2013). 
Now, we consider data about the lifetime sexual partners for both males and females reported in the Health and Sexual Habits Survey in Spain [11] as can be seen in Figure 2. The asymmetry in the behavior of males and females have to be taken into account in the construction of the network.
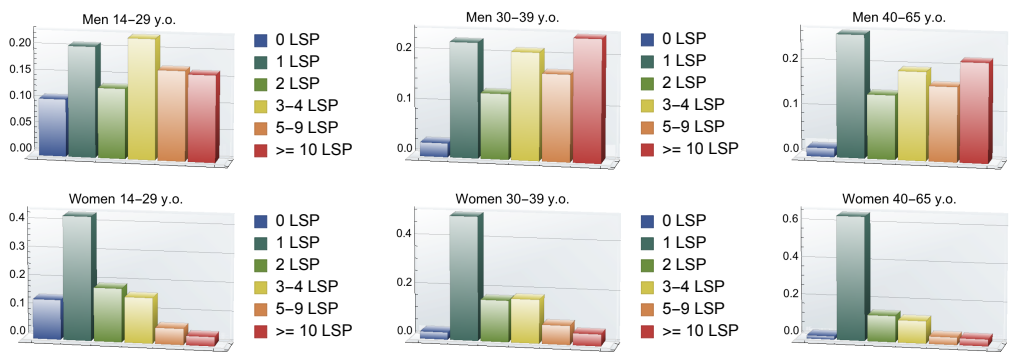

0 LSP 1 LSP
2 LSP $3-4$ LSP 5-9 LSP
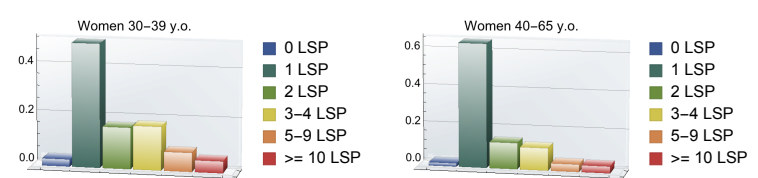

Figure 2. Number of lifetime sexual partners (LSP) for men and woman per age group in Spain (year 2003). The age groups are 14-29, 30-39 and 40-65.

The estimated percentage of homosexual men is 3.88\% [11]. The situation for the homosexual men population is different of the one shown in Figure 2, because the average number of sexual partners is estimated in 39 regardless of age, but this number increases with age with a peak of 59 in the 40-49 age-group [14].

A difficulty arises because we have little information about the number of sexual contacts in homosexual women subpopulation. In a personal communication by Dr. Mireia Díaz from the Catalan Institute of Oncology (IDIBELL) we were informed that HPV hardly spreads among homosexual women, and almost all homosexual men, sometimes in their lives, had a woman partner. Consequently, we have simulated these connections by assigning a contact to every man in the homosexual subpopulation with woman with 5 partners or more. This is done according to the assortativity principle, that is, people use to join with people with similar habits.

Taking into account the above premises, for the average number of LSP of men $k$ and for each one of the $N$ individuals (nodes) in the network

1. Using the demographical information, we assign randomly the sex (man/woman) and age.

2. We label $3.88 \%$ of men as homosexual men, randomly.

3. Depending on the age and gender, and using the data about LSP for men and women, we assign randomly the number of LSP he/she is going to have.

4. We assign randomly LSP to homosexual men depending on their age following the report [14].

The above algorithm determines the sex, age and number of LSP of each node. Now, we have to build the network that matches with the LSP of the nodes.

1. Separate men and women nodes into two groups. 
2. Order women in descendant order of LSP.

3. For each woman node:

(a) we pair her with as much heterosexual men as the number of LSP she has. Among all the possibilities to pair we try to choose those men with similar number of LSP.

4. For each homosexual men node:

(a) we pair him with as much homosexual men as the number of LSP he has.

(b) Assign to every homosexual men a woman with 5 partners or more.

The procedure to pair heterosexual partners is the same we use to pair homosexual partners. In Figure 3 we can see a small LSP network. Details about how to build the heterosexual networks can be found in [4].

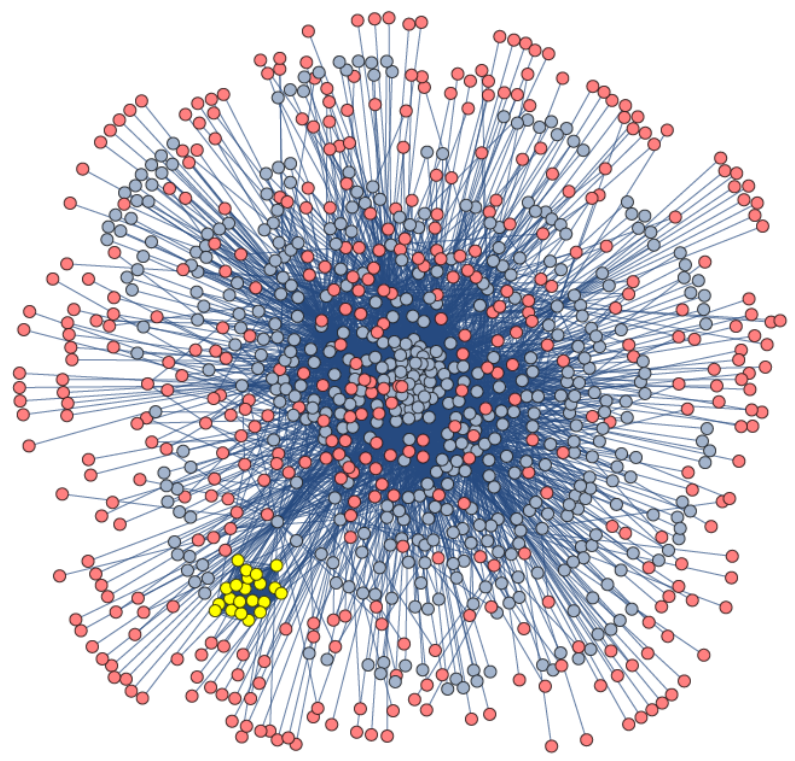

Figure 3. LSP network for 1000 individuals: blue dots correspond to men, pink to women and yellow to homosexual men.

\section{HPV prevalence data}

Data provided by [2] allow us to determine an estimation of the prevalence of the HPV. Then, we divide the data of infectious women into the age groups $18-29,30-39$ and $40-65$, the same age groups as those given by the Sexual Behaviour Habits Survey [11] and used to build the LSP networks.

Taking into account that an infected individual with high risk HPV may develop precancerous lesions and, eventually, cancer, and that an infected individual 
with low risk HPV may develop genital warts, we say that an individual is HRinfected if he/she has been infected by a high risk HPV. That individual may also be infected by a low risk HPV (co-infection). Analogously, an individual is LR-infected if he/she has been infected by a low risk HPV. That individual may also be infected by a high risk HPV (co-infection). Then, the percentage of women HR- and LR-infected per the aforementioned group ages, are collected in Table 1.

Table 1. Prevalence of HR- and LR-infected women per age groups. Mean and $95 \%$ confidence intervals.

\begin{tabular}{c|cc}
\hline Women & HR-infected & LR-infected \\
\hline $18-29$ y.o. & $24.10 \%,[21.33 \%, 26.98 \%]$ & $6.36 \%,[4.71 \%, 8.07 \%]$ \\
$30-39$ y.o. & $11.01 \%,[7.54 \%, 15.09 \%]$ & $1.26 \%,[0.0 \%, 3.14 \%]$ \\
$40-64$ y.o. & $5.96 \%,[4.29 \%, 7.8 \%]$ & $2.37 \%,[1.22 \%, 3.68 \%]$ \\
\hline $18-64$ y.o. & $16.23 \%,[14.52 \%, 17.97 \%]$ & $4.41 \%,[3.42 \%, 5.45 \%]$ \\
\hline
\end{tabular}

\section{HPV dynamics on a LSP network}

Over the LSP networks, we introduce the transmission dynamics of the HPV. Thus, non-infected individuals may get infected if they have sexual intercourses with HR- or LR-infected sexual partners. Being infected, the individuals may infect other susceptible sexual partners. After a period of time depending on the type of infection (HR or LR), the individuals recover and move from the contagious state to susceptible state, clearing the infection.

The above description leads to consider the following model parameters that determine the model behavior. These parameters are:

- Global probabilities in order to determine if a LSP produces a contagion in the current time step per age group $14-17,18-29,30-39$ and $40-65$. Taking into account that the edges that represent LSP are fixed and permanent, we need to modulate the possibility of contagion in a certain period. Then, we include the model parameters $T_{0}, T_{1}, T_{2}$ and $T_{3}$ to modulate these contagion in every month for group ages $14-17,18-29$, $30-39$ and $40-65$, respectively (4 parameters).

- Average time an individual infected by a high (low) risk HPV clears the infection and recovers (2 parameters).

- Probability that a woman (man) infected of high (low) risk HPV transmits it to his/her partner in a sexual intercourse (4 parameters).

Also, we should recall that, for LSP network building, we need to provide the average number of men LSP $k$. Then, we have a total of 11 model parameters to be determined.

For the simulations we are going to show hereinafter, the networks will have 200000 nodes and the temporal step used is defined as a month.

Prepared using sagej.cls 
Hence, we have implemented in $\mathrm{C}++$ a simulator that, given the above described model parameters, it builds a LSP network and performs a simulation of the transmission dynamics of high and low risk HPV.

Note that the transmission parameters involve certain probabilities. Then, in order to see if a contagion has been carried out by a sexual intercourse, we simulate this by generating a random number and checking if it is less than the corresponding threshold given by the model transmission parameters. Therefore, the randomness is included into the model in a natural way producing some uncertainty on the model output that has to be quantified.

After all the above considerations, now, the goal is, assuming that we are in a stable situation, to calibrate the model parameters in such a way that the model output related to women HR and LR prevalence is as close as possible to the data in Table 1.

\section{Preparing the distributed computing environment for model calibration}

\section{Sisifo distributed computing environment}

Sisifo is a client-server based system designed to allow a problem to be solved using distributed computation. Sisifo is able to assign tasks to a set of personal computers (PCs), wait for the tasks to complete and collect the results for further analysis. Sisifo is made with simplicity as main aim, giving as a result a system that requires almost no maintenance, needs very little configuration time, and can be deployed in just a couple of hours.

The Sisifo Server keeps listening for request of the clients. The Server has stored one or more executors, a set of problems to be solved in the Problem files folder, and the solutions sent in the Result files folder.

The Sisifo Client is a program stored in one or several PCs that connects to the server, and asks for a work packet. This work packet is composed of two elements: a text file containing the model parameter values and the simulator executable file. The Client, once the work packet is received, executes a simulation using the model parameters stored in the text file. When the simulation finishes, a solution file is generated, returned to the server and dropped in the Results files folder.

More details about how Sisifo works can be found in [9].

\section{PSO-based calibration procedure}

As we mentioned in the Introduction, we used in the past Sisifo to calibrate models using exhaustive searching. With this method, we needed to perform a lot of simulations and the best obtained fitting was not always as closed as desired. For instance, in [9] 145000 simulations were performed achieving the equivalent of more than three years of computing time in just five weeks. However, even

Prepared using sagej.cls 
eventually we could find a feasible solution, most of the simulations were useless, far from being feasible solutions.

For this reason, we consider the implementation of a version of PSO optimization algorithm adapted to Sisifo computing environment. To do that, first, we recall the rPSO algorithm appearing in [15] applied to the optimization of a function $F$.

Step 1. Initialization.

- Initialize $N$ particles $p_{1}, \ldots, p_{N}$ chosen randomly in the parameter space.

- Initialize randomly their velocities $v_{1}, \ldots, v_{N}$.

- Evaluate the fitness of all the particles $F\left(p_{1}\right), \ldots, F\left(p_{N}\right)$.

- Define the individual best fitness as $p_{i}^{\text {best }}=p_{i}, i=1, \ldots, N$ and the global best fitness $p_{\text {global }}^{\text {best }}$ as the $p_{i}^{\text {best }}$ which fitness is optimum.

Step 2. Modify the particle velocities based on the previous individual best and global best positions:

$$
v_{i}^{\text {new }}=\omega v_{i}+\psi_{1}\left(p_{i}^{\text {best }}-p_{i}\right)+\psi_{2}\left(p_{\text {global }}^{\text {best }}-p_{i}\right), i=1, \ldots N,
$$

where $\omega$ is a random value in $\left[\frac{1}{4}, \frac{3}{4}\right], \psi_{1}$ is the exploitation rate and $\psi_{2}$ is the exploration rate.

Step 3. Update the particle locations: $p_{i}=p_{i}+v_{i}^{\text {new }}, i=1, \ldots N$.

Step 4. Evaluate the fitness of all the particles $F\left(p_{1}\right), \ldots, F\left(p_{N}\right)$. Go to Step 2 .

The above algorithm can be adapted to Sisifo computing environment if, using the Sisifo Server, the computation of the fitness of the particles is distributed among the Sisifo Clients.

However, in a typical PSO procedure, including rPSO, the set of particles is updated once the fitness of all the particles have been calculated. This means that, until all the fitnesses have not been evaluated and Step 4 is not completely finished, the particles cannot be updated and new evaluations cannot be performed. Then, in every iteration of rPSO, scenarios where some Sisifo Clients have finished their evaluations and are idle while other Sisifo Clients are still performing their evaluations are usual. In these scenarios, we have an under-use of the Sisifo system.

In order to avoid the under-use system drawback, we propose the implementation of an asynchronous version of rPSO in such a way that when the fitness of a particle has been evaluated (Step 4), this particle is updated (Step 2 and 3) without waiting for the evaluation of the remainder particles, considering the current existing global best and its individual best particles. This way, we modify rPSO algorithm parallelizing Steps 2, 3, 4 and sharing the updates of the global best particle.

Prepared using sagej.cls 
The proposed asynchronous version of rPSO has been implemented in Mathematica [16] and it is shown in Figure 4. When the procedure starts running, with the initialization of the particles (Step 1) we create their corresponding problem files in the Problem files folder. The Sisifo Server detects new problem files and distribute them among free Sisifo Clients. These clients carry out the simulations. When a Sisifo Client ends its task, a results file is generated and sent to the Sisifo Server that drops it in the Result files folder. Every time a new results file appear in the Results files folder, the PSO-based calibration procedure, that is, the asynchronous rPSO, in Step 4, reads the data from the results file and calculates the fitness (distance from the model output to the data in Table 1). Then, updates the velocity taking into account the current existing global best and its individual best particles (Step 2), updates the particle (Step 3) and with the new model parameters creates a new problem file in the Problem files folder. And so on.

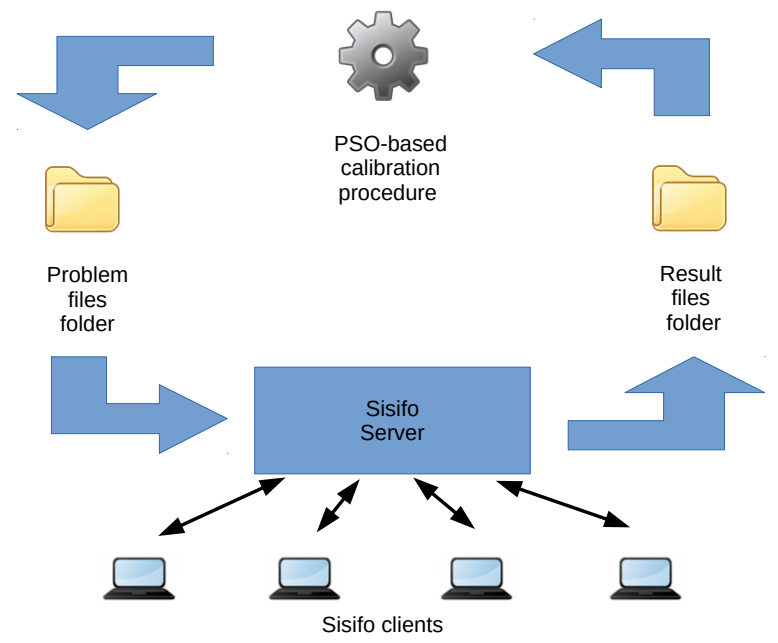

Figure 4. Scheme of the PSO-based calibration procedure (asynchronous rPSO) in the Sisifo distributed computing environment.

Even though we have to perform several attempts of the PSO-based calibration procedure in a typical fitting problem, we are able to determine if the calibration is acceptable or not computing around 2000 simulations in a couple of days. Comparing with the time and number of simulations used in [9], 145000 during five weeks, we can have an idea of the saving of resources involved.

\section{Results}

Once the model has been calibrated, due to the intrinsic randomness in the LSP networks building and the evolution of the HPV infection, we carry out 100 simulations and calculate the mean and the $95 \%$ confidence interval (CI95\%) in each time step of the 100 model outputs. Then, this is compared with the 
data mean and CI95\% in Table 1. The results for the age group $18-64$ years old can be seen in Figure 5.

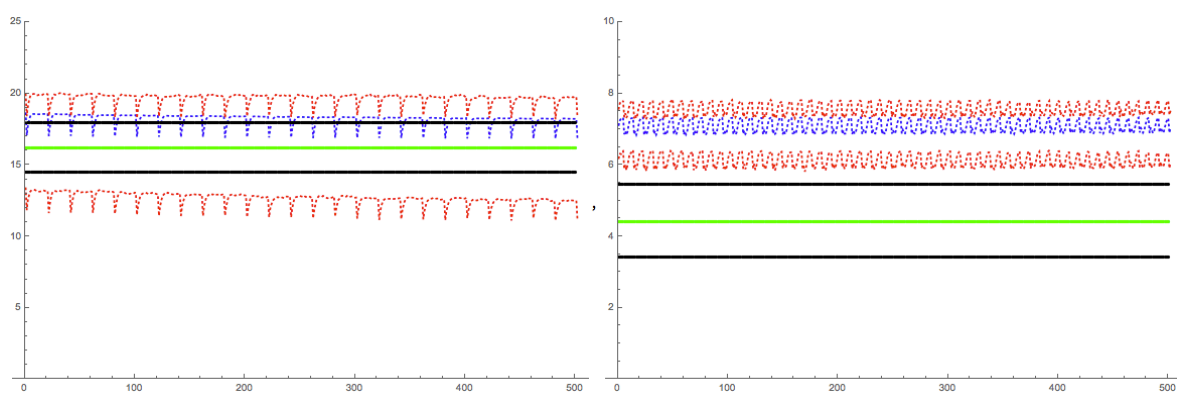

Figure 5. Mean and $\mathrm{Cl} 95 \%$ of the percentage of model output for HR-infected (left) and for LR-infected (right), compared with their data counterparts, for women in the age group 18-64 years old. The blue points represent the mean of the model output and the red points its $\mathrm{Cl} 95 \%$. The green line is the mean of data and the black lines its $\mathrm{Cl}$. $55 \%$.

Numerically, the model output mean and CI95\% for women HR-infected in the age group $18-64$ years old are $18.21 \%$, CI95\% [12.66\%,19.68\%]. And for LRinfected, $7.08 \%$, CI95\% [6.13\%, 7.56\%].

Looking at the Figure 5 and data in Table 1, we should note that the calibration is fair enough: for women HR-infected data and model uncertainty (CI95\%) almost match; for women LR-infected do not match, however they are very near. Moreover, in Figure 6 we present a comparison as the one in Figure 5, but for age group $18-29$. The calibration is also performed for the remainder age groups $30-39$ and $40-65$.

\section{HR-infected}

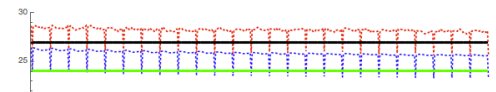

18-29 у.о.

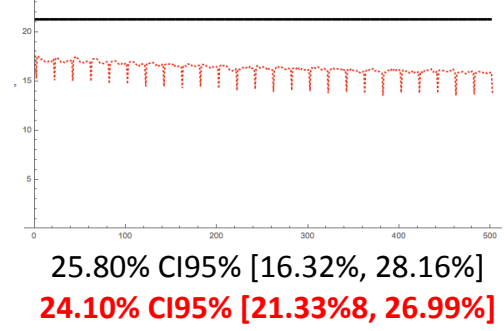

$\underline{\text { LR-infected }}$
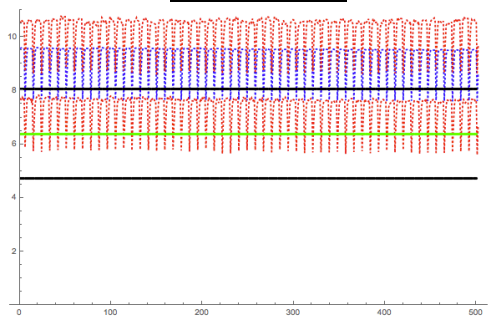

$9.21 \% \mathrm{Cl} 195 \%[7.33 \%, 10.27 \%]$

$6.36 \% \mathrm{Cl} 95 \%[4.71 \%, 8.07 \%]$

Figure 6. As in Figure 5, we present the model output mean and C195\% compared with their data counterparts per women age group $18-29$. The blue points represent the mean of the model output and the red points its $\mathrm{C195 \%}$. The green line is the mean of data and the black lines its $\mathrm{Cl} 95 \%$. At the bottom of the graphs we have the model output mean and $\mathrm{Cl} 95 \%$ in black and data mean and $\mathrm{Cl} 95 \%$ in bold red. 
In general, the model calibration is fairly good and reproduces accurately the data presented in [2], given that the complexity of this epidemic problem.

\section{About the model parameters obtained in the calibration}

Apart from the calibration, it is usual to check if the model also satisfies some side effects in the dynamics of the HPV, in order to present a model as much credible as possible.

Thus, let us examine the credibility of the calibrated model parameters comparing them with some values appeared in the literature. These model parameters obtained are:

- Average number of LSP of men: 8.8. This value is consistent with the one given in [14], where 8 is stated as the average number of LSP.

- Average duration of an infection due to high and low risk HPV: 1.56 and 0.66 years, respectively. These values are similar to the ones given in [17], 1.2 and 0.7 years, for the infection duration of HPV 16/18 (high risk) and $6 / 11$ (low risk), respectively.

- Probability a woman/man infected of HPV transmits the infection to a sexual partner: 0.54 and 0.73 for high risk HPV and 0.47 and 0.62 for low risk HPV, respectively. In [17], the authors present the global values 0.7 and 0.8 for all the types.

\section{Conclusion}

In the recent years, we started to work with large network models, mainly applied to study the transmission dynamics of infectious diseases. Then we needed resources, not only computers but also ad-hoc software. Hence we decided to design a distributed computing environment called Sisifo that allowed to perform a large amount of simulations in a reasonable time taking full advantage of the available computers and all their processors.

However, for calibration, we had to perform a lot of simulations in order to find a fairly good calibration. And we thought in the possibility to adapt well-known optimization algorithms to our environment.

Our first approach has been to adapt a random version of the Particle Swarm Optimization called rPSO to calibrate a HPV transmission dynamics model on LSP networks. The results have been better in terms of computation time and number of performed simulations compared with the exhaustive searching strategy we have been used so far.

Moreover, the model calibration also satisfies some other expected features described in the literature, as the average number of LSP of men, the time an individual clears the infection and the transmission parameters, which abounds in the credibility of model.

Nevertheless, this is only a part of the general problem of studying the transmission dynamics of infectious diseases: once the model has been calibrated,

Prepared using sagej.cls 
we are able to forecast the evolution of the spread of the infectious diseases and to study the implementation of vaccination strategies. As a consequence, it is possible to calculate the economical cost of the application of a vaccination schedule comparing the prediction of the model in this vaccination scenario with the model prediction with no vaccination using the calibrated model parameters.

In the future, we will introduce in Sisifo other optimization algorithms and apply all this developed infrastructure to customize treatments for diabetic patients using and calibrating models describing the patient's glucose dynamics.

\section{Author Biographies}

Luis Acedo is a researcher at the Institute for Multidisciplinary Mathematics of the Universitat Politècnica de Valéncia in Valencia, Spain. He is $\mathrm{PhD}$ in fundamental physics at the University of Extremadura in Badajoz, Spain. His current research interests are interdisciplinary, including mathematical modelling of infectious diseases, epidemiology, networks and gravitational physics. He has also organized ten international conferences on mathematical modelling.

Clara Burgos is a $\mathrm{PhD}$ student in the Universitary Institute of Multidisciplinary Mathematics in the Universitat Politècnica de Valéncia. She is working in modeling the spread of infectious diseases.

José-Ignacio Hidalgo is $\mathrm{PhD}$ in physics and professor in the Department of Computer Architecture and Automation of the Universidad Complutense de Madrid. His research is focused in evolutionary bio-inspired optimization algorithms and customization of treatments to diabetic patients.

Víctor Sánchez-Alonso works in a Spanish information technology and defense systems company and is a $\mathrm{PhD}$ student in the Universitary Institute of Multidisciplinary Mathematics in the Universitat Politècnica de Valéncia. His research interest is computational epidemiology models.

Rafael J. Villanueva is $\mathrm{PhD}$ in Applied Mathematics and professor in the Universitat Politécnica de Valencia. His main area of interest is modeling the transmission dynamics of the infectious diseases. Also, he is interested in the uncertainty of the real data and their effect on the model predictions.

Javier Villanueva-Oller is a $\mathrm{PhD}$ in Applied Mathematics, professor in the University Rey Juan Carlos and also works in a Spanish information technology and defense systems company. His main interest is network modeling of the transmission dynamics of infectious diseases.

\section{Declaration of conflicting interests}

The authors declares that there is no conflict of interest.

\section{Funding}

This work has been partially supported by the Ministerio de Economía y Competitividad grants MTM2013-41765-P and TIN 2014-54806-R.

Prepared using sagej.cls 


\section{References}

[1] What is HPV? URL https://www.cdc.gov/hpv/parents/whatishpv . html.

[2] Castellsagué X, Iftner $\mathrm{T}$, Roura $\mathrm{E}$ et al. Prevalence and genotype distribution of human papillomavirus infection of the cervix in Spain: The CLEOPATRE study. Journal of Medical Virology 2012; 84(6): 947-956. DOI:10.1002/jmv.23282. URL https://doi.org/10.1002\%2Fjmv. 23282.

[3] Fairley C, Hocking J, Chen M et al. Rapid decline in warts after national quadrivalent HPV vaccine program. 25th International Papillomavirus Conference: Clinical \& Educational Workshop, Malmö, Sweden, May 8-14, 2009, pp. Abstract O-29.02.

[4] Acedo L, Martí R, Palmi F et al. Building lifetime heterosexual partner networks. In Jódar L, de la Poza E and Acedo L (eds.) Modeling human behavior. Individuals and organizations, chapter 19. Nova publishers, 2017. pp. 235-251.

[5] Acedo L, Moraño JA, Villanueva RJ et al. Using random networks to study the dynamics of respiratory syncytial virus (RSV) in the Spanish region of Valencia. Mathematical and Computer Modelling 2011; 54(7-8): 1650-1654. DOI:10.1016/j.mcm.2010.11.068. URL https://doi.org/10. $1016 \% 2 \mathrm{Fj}$.mcm . 2010.11.068.

[6] Acedo L, Lamprianidou E, Moraño JA et al. Firing patterns in a random network cellular automata model of the brain. Physica A: Statistical Mechanics and its Applications 2015; 435: 111-119. DOI:10.1016/j.physa. 2015.05.017. URL https://doi.org/10.1016\%2Fj . physa.2015.05.017.

[7] González-Parra G, Villanueva RJ, Ruiz-Baragaño J et al. Modelling influenza A(H1N1) 2009 epidemics using a random network in a distributed computing environment. Acta Tropica 2015; 143: 29-35. DOI:10. 1016/j.actatropica.2014.12.008. URL https://doi.org/10.1016\%2Fj . actatropica.2014.12.008.

[8] Sisyphus (Sisifo in Spanish). URL https://en.wikipedia.org/wiki/ Sisyphus.

[9] Villanueva-Oller J, Acedo L, Moraño JA et al. Epidemic random network simulations in a distributed computing environment. Abstract and Applied Analysis 2013; 2013: 1-10. DOI:10.1155/2013/462801. URL https: //doi.org/10.1155\%2F2013\%2F462801.

[10] Cortés JC, Colmenar JM, Hidalgo JI et al. Modeling and predicting the Spanish bachillerato academic results over the next few years using a random network model. Physica A: Statistical Mechanics and its Applications 2016; 442: 36-49. DOI:10.1016/j.physa.2015.08.032. URL https://doi .org/10.1016\%2Fj . physa. 2015.08.032.

Prepared using sagej.cls 
[11] Encuesta de salud y hábitos sexuales 2003 (Health and sexual habits survey). Instituto Nacional de Estadística, 2003. URL http://www.ine. es/.

[12] Community of Valencia. URL https://en.wikipedia.org/wiki/ Valencian_Community.

[13] Portal estadístico de la Generalitat Valenciana (Statistical portal of the government of the Community of Valencia), 2013. URL http://www. pegv . gva.es/.

[14] Estudio de conducta sexual entre homosexuales (Study of sexual behavior among homosexuals). Technical report, Durex, 2002.

[15] Khemka N and Jacob C. Exploratory toolkit for evolutionary and swarmbased optimization. The Mathematica Journal 2010; 11(3): 376-391. DOI: 10.3888/tmj.11.3-5. URL https://doi.org/10.3888\%2Ftmj.11.3-5.

[16] Mathematica. URL http://www.wolfram.com/mathematica/.

[17] Elbasha EH, Dasbach EJ and Insinga RP. Model for assessing human papillomavirus vaccination strategies. Emerging Infectious Diseases 2007; 13(1): 28-41. DOI:10.3201/eid1301.060438. URL https://doi.org/10. $3201 \% 2 \mathrm{Feid} 1301.060438$.

Prepared using sagej.cls 